\title{
Dynamical Magnetoelectric Coupling in Multiferroic $\mathrm{BiFeO}_{3}$
}

\author{
J. A. Bartkowska
}

Received: 31 March 2010 / Accepted: 13 January 2011 / Published online: 4 February 2011

C The Author(s) 2011. This article is published with open access at Springerlink.com

\begin{abstract}
In this study, the magnon excitations in multiferroic $\mathrm{BiFeO}_{3}(\mathrm{BFO})$ have been discussed. The studies are based on the spin wave theory and Katsura's model. The influence of the spin wave excitations on the terahertz absorption is discussed. The antiferromagnetic and ferroelectric interactions in multiferroic BFO were included using an effective fermion Hamiltonian. This Hamiltonian is bosonized and diagonalized, using Holstein-Primakoff and Bogoliubov transformations, respectively. An effective boson Hamiltonian is diagonalized to determine the excitation energy of the spin wave. The results obtained in this study are in qualitative agreement with the experimental data.
\end{abstract}

Keywords Magnetoelectric coupling - Magnons · Multiferroics · Spin wave · Spiral magnetic ordering

\section{Introduction}

In recent years, multiferroics have received significant attention in the field of material science. Multiferroics are compounds that possess two or three forms of primary ferroic properties: ferroelectricity, ferromagnetism, and ferroelasticity.

The current trend is to focus on ferroelectric-ferromagnetism. Magnetic ferroelectrics may constitute the most interesting type of multiferroics because they may exhibit an unusually strong, so-called magnetoelectric coupling of magnetic and electric properties. Owing to the interaction between the electric polarization and magnetic polarization, the coexistence of ferroelectricity and ferromagnetism has the possibility to

\footnotetext{
J. A. Bartkowska ( $\varangle)$

Department of Materials Science, Faculty of Computer Science and Material Science,

Silesian University, 12 Żytnia St., Sosnowiec 41-200, Poland

e-mail: joanna.bartkowska@us.edu.pl
} 
exhibit the magnetoelectric effect, that is, electric polarization is changed by an external magnetic field, or magnetic polarization is changed by an external electric field $[1,2]$.

Magnetoelectric materials have opportunities for potential applications in information storage, the emerging field of spintronics, and sensors. Other potential applications include multiple state memory elements, in which data are stored in both the electric and magnetic polarizations. In addition to the potential applications, the fundamental physics of magnetoelectric materials is very fascinating.

One of the basic multiferroic materials is bismuth ferrite, $\mathrm{BiFeO}_{3}$ (BFO). This compound possesses a rhombohedrally distorted bimolecular perovskite unit cell (space group $R 3 c$ ) with the parameters (in a hexagonal system) $a_{\text {hex }}=0.5571 \mathrm{~nm}$ and $c_{\text {hex }}=$ $1.3858 \mathrm{~nm}$ [3]. Bismuth ferrite is an antiferromagnetic ferroelectric with an antiferromagnetic Néel temperature $T_{\mathrm{N}} \sim 643 \mathrm{~K}$ and a ferroelectric Curie temperature $T_{\mathrm{C}} \sim$ $1103 \mathrm{~K}$ [4]. The BFO is a very rare example of multiferroic materials with both the magnetic and ferroelectric ordering temperatures above room temperature.

The primary magnetic order in BFO is G-type antiferromagnetism, which is subjected to a modulation associated with a spiral of periodicity of approximately $\lambda_{\mathrm{S}}=62 \mathrm{~nm}$ [3-5]. The BFO exhibits a magnetoelectric effect, and recently, Raman scattering spectroscopy was used to study the magnon spectra of this material [6-8]. Bismuth ferrite also exhibits an infrared absorption. A detailed study of the terahertz spectrum of BFO revealed that infrared absorption is observed $[9,10]$, and this fact is not explained by the theory.

In this article, the relation between infrared absorption and the characteristic excitations of the spin wave, i.e., magnons, is discussed.

\section{Spin Wave Hamiltonian}

The model of Katsura et al. [11] is used to describe the spin wave excitations. In this article, the author assumed that an effective Hamiltonian of a multiferroic material contains the following three kinds of interactions: Heisenberg interactions $\left(H_{\mathrm{ex}}\right)$, Dzyaloshinskii-Moriya interactions $\left(H_{\mathrm{DM}}\right)$, and the interactions associated with the dynamics of the lattice $\left(H_{\text {kin }}\right)$.

An effective Hamiltonian describing the coupling between the spins and the atomic displacement $\mathbf{u}_{i}$, which represents the electric polarization including the displacements of an electronic cloud, is given as

$$
H=H_{\mathrm{ex}}+H_{\mathrm{DM}}+H_{\mathrm{kin}},
$$

with

$$
\begin{aligned}
H_{\mathrm{ex}} & =-\sum_{\langle i j\rangle} J \mathbf{S}_{i} \cdot \mathbf{S}_{j}, \\
H_{\mathrm{DM}} & =-\lambda \sum_{i} \mathbf{u}_{i} \cdot\left[\mathbf{e}_{z} \times\left(\mathbf{S}_{i} \times \mathbf{S}_{i+1}\right)\right],
\end{aligned}
$$




$$
H_{\text {kin }}=\sum_{i}\left(\frac{\kappa}{2} \mathbf{u}_{i}^{2}+\frac{1}{2 M} \mathbf{P}_{i}^{2}\right)
$$

In the above Hamiltonian, Eq. 1, $H_{\mathrm{ex}}$ is the Heisenberg exchange Hamiltonian for the nearest-neighbor spin interaction $\mathbf{S}_{i}$ and $\mathbf{S}_{i+1}$ of the host multiferroic material; the notation $\langle i j\rangle$ denotes the nearest-neighbor bonds. The exchange parameter $J$ between the nearest-neighbor spins is considered to be constant. The term $H_{\mathrm{DM}}$ represents the Dzyaloshinskii-Moriya interaction, the spin-lattice interaction strength is denoted by $\lambda$, and $\mathbf{e}_{z}$ is the unit vector. The dynamics of the lattice field is included in $H_{\text {kin }}$ where $\mathbf{u}_{i}$ is the atomic displacement at site $i$ and the canonically conjugate momentum is $\mathbf{P}_{i}$. In $H_{\text {kin }}, \kappa$ and $M$ are the spring constant and the effective mass of $\mathbf{u}_{i}$, respectively.

As is well known, bismuth ferrite is an antiferromagnetic ferroelectric material. An antiferromagnetic interaction acts to the anti-align neighboring spins; therefore, in an antiferromagnetic case it is assumed that the lattice is divided into two interpenetrating sublattices, in which spins have the opposite directions, e.g., A and B sublattices. Spins $i$ and $j$ belong to A and B sublattices, respectively.

Minimizing the Hamiltonian, Eq. 1, yields the condition of the atomic displacement and the local spin configuration with the following form:

$$
\mathbf{u}_{i}=\frac{\lambda}{\kappa} \mathbf{e}_{z} \times\left(\mathbf{S}_{i} \times \mathbf{S}_{i+1}\right)
$$

Assuming the one-dimensional spin chain along the $z$-axis, and $z x$ spiral spins along the chain, that is, $\mathbf{S}_{i}=S(\sin i Q, 0, \cos i Q)$, Eq. 5 leads to the microscopic uniform lattice displacement along the $x$-direction,

$$
\mathbf{u}_{x}=-\frac{\lambda S^{2}}{\kappa} \sin Q \mathbf{e}_{x},
$$

where $Q$ is the spiral wave vector.

\subsection{Spin Wave Excitations-Magnons}

One standard and simple theory used for calculating the magnetization, polarization, and energy of the magnetically and electrically ordered phases is the linear spin wave theory $[12,13]$. In this study, just the linear spin wave theory is applied to the model of magnon excitations.

The one-dimensional spin chain along the $z$-axis can be considered. For the $z x$ spiral spins, it is convenient to rotate the quantum projection axis of the spins at each site along its classical direction. It is convenient to rotate the spins locally, at each site, along its classical direction $\hat{S}_{i}^{z}$, and therefore, use the following transformations of spin operators: 


$$
\begin{aligned}
& \mathbf{S}_{i}^{x}=\hat{S}_{i}^{x} \cos i Q+\hat{S}_{i}^{z} \sin i Q, \\
& \mathbf{S}_{i}^{y}=\hat{S}_{i}^{y}, \\
& \mathbf{S}_{i}^{x}=-\hat{S}_{i}^{x} \sin i Q+\hat{S}_{i}^{z} \cos i Q,
\end{aligned}
$$

to transform the Hamiltonian, expressed by Eq. 1 .

After this transformation and disregarding the higher-order terms of the interplay between the spins, the components of the microscopic Hamiltonian, namely, Eqs. 2-4, are given by

$$
\begin{aligned}
H_{\mathrm{ex}}= & \sum_{\langle i j\rangle} J\left[\cos (i Q-j Q)\left(\hat{S}_{i}^{x} \hat{S}_{j}^{x}+\hat{S}_{i}^{z} \hat{S}_{j}^{z}\right)\right. \\
& \left.+\sin (i Q-j Q)\left(\hat{S}_{i}^{z} \hat{S}_{j}^{x}-\hat{S}_{i}^{y} \hat{S}_{j}^{y}\right)+\hat{S}_{i}^{y} \hat{S}_{j}^{y}\right], \\
H_{\mathrm{DM}}= & -\lambda \sum_{i} u_{i}^{x} \frac{1}{2}\left\{\cos Q\left[\hat{S}_{i}^{x} \hat{S}_{i+1}^{z}-\hat{S}_{i+1}^{x} \hat{S}_{i}^{z}+\hat{S}_{i+1}^{z} \hat{S}_{i}^{x}-\hat{S}_{i}^{z} \hat{S}_{i+1}^{x}\right]\right. \\
& +\cos (2 i Q+Q)\left[{ }_{i}^{x} \hat{S}_{i+1}^{z}-\hat{S}_{i+1}^{x} \hat{S}_{i}^{z}-\hat{S}_{i+1}^{z} \hat{S}_{i}^{x}+\hat{S}_{i}^{z} \hat{S}_{i+1}^{x}\right] \\
& -\sin (Q)\left[\hat{S}_{i+1}^{x} \hat{S}_{i}^{x}+\hat{S}_{i}^{z} \hat{S}_{i+1}^{z}+\hat{S}_{i}^{x} \hat{S}_{i+1}^{x}+\hat{S}_{i+1}^{z} \hat{S}_{i}^{z}\right] \\
& \left.+\sin (2 i Q+Q)\left[\hat{S}_{i+1}^{x} \hat{S}_{i}^{x}+\hat{S}_{i}^{z} \hat{S}_{i+1}^{z}-\hat{S}_{i}^{x} \hat{S}_{i+1}^{x}-\hat{S}_{i+1}^{z} \hat{S}_{i}^{z}\right]\right\} \\
H_{\text {kin }}= & \sum_{i}\left(\frac{\kappa}{2} \mathbf{u}_{i}^{2}+\frac{1}{2 M} \mathbf{P}_{i}^{2}\right),
\end{aligned}
$$

where $\hat{S}_{i}^{x}, \hat{S}_{i}^{y}$, and $\hat{S}_{i}^{z}$ are the spin operators.

The spin wave excitations, i.e., magnons, are bosons; therefore, an effective Hamiltonian, Eqs. 8-10, should be bosonized. Using the standard Holstein-Primakoff representation [14] for the spin operators, and the Fourier transform of the boson operators, the microscopic Hamiltonian for the spiral multiferroic can be bosonized. As was noticed by Holstein and Primakoff [14], the operators, $a_{i}, a_{i}^{+}, b_{j}, b_{j}^{+}$, are to be considered as matrices with infinite dimensions in order to satisfy the commutation rule, $a_{i} a_{i}^{+}-a_{i}^{+} a_{i}=1$. This is very useful in simplifying the mathematics and makes the spin wave particles obey Bose statistics. Holstein-Primakoff transformations of spin operators to boson operators on each sublattice (A and B) are

$$
\begin{aligned}
S_{i}^{+} & =\sqrt{2 S-a_{i}^{+} a_{i}} a_{i}, \\
S_{i}^{+} & =a_{i}^{+} \sqrt{2 S-a_{i}^{+} a_{i}}, \\
S_{i}^{z} & =S-a_{i}^{+} a_{i}, \\
S_{j}^{+} & =b_{j}^{+} \sqrt{2 S-b_{j}^{+} b_{j}}, \\
S_{j}^{+} & =\sqrt{2 S-b_{j}^{+} b_{j}} b_{j},
\end{aligned}
$$




$$
S_{j}^{z}=-S+b_{j}^{+} b_{j}
$$

with

$$
\hat{S}_{i}^{x}=\frac{1}{2}\left(S_{i}^{+}+S_{i}^{-}\right), \quad \hat{S}_{i}^{y}=-\frac{i}{2}\left(S_{i}^{+}-S_{i}^{-}\right),
$$

where $a_{i}^{+}$and $b_{j}^{+}$denote boson creation operators on sublattices A and B, respectively, and $a_{i}, b_{j}$ denote boson annihilation operators on sublattices A and $\mathrm{B}$, respectively. Boson creation and annihilation operators obey the usual boson commutation relations.

The Fourier transformation was introduced in the following form:

$$
\begin{aligned}
& a_{i}^{+}=\sum_{q} \frac{1}{\sqrt{N / 2}} a_{q}^{+} \mathrm{e}^{\mathrm{i} q r_{i}}, \quad a_{i}=\sum_{q} \frac{1}{\sqrt{N / 2}} a_{q} \mathrm{e}^{-\mathrm{i} q r_{i}}, \\
& b_{j}^{+}=\sum_{q} \frac{1}{\sqrt{N / 2}} b_{q} \mathrm{e}^{-\mathrm{i} q r_{j}}, \quad b_{j}=\sum_{q} \frac{1}{\sqrt{N / 2}} b_{q}^{+} \mathrm{e}^{\mathrm{i} q r_{j}} .
\end{aligned}
$$

Now, if the bilinear terms are only respected in spin wave operators, the effective Hamiltonian, Eqs. 8-10, can be approximated by

$$
H=E_{0}+\sum_{q} A(q)\left(a_{q}^{+} a_{q}+b_{q}^{+} b_{q}\right)+\left(a_{q} a_{-q}+b_{q}^{+} b_{-q}^{+}\right)
$$

with

$$
\begin{aligned}
E_{0} & =N\left[J S^{2} \cos Q-\frac{\kappa}{2}\left|u_{0}^{x}\right|^{2}-\frac{1}{2 M} P^{2}\right], \\
A(q) & =-J\left[\cos Q+\frac{1}{2}(1+\cos Q) \cos q\right]+\frac{\lambda^{2} S^{2} \sin ^{2} Q}{2 \kappa}(2-\cos q), \\
B(q) & =\frac{J}{4}(1-\cos Q) \cos q-\frac{\lambda^{2} S^{2} \sin ^{2} Q}{4 \kappa} \cos q,
\end{aligned}
$$

where $N$ is the number of sites on the lattice and $q$ is the magnon wave vector.

The microscopic Hamiltonian is not diagonal in the boson operators. Following standard linear spin wave theory, the Hamiltonian can be diagonalized using a Bogoliubov transformation, assuming a spiral ordering of the spins in the lattice so that the relative angle between the two nearest-neighbor spins is $Q$. Taking the Bogoliubov transformation into account

$$
\begin{array}{ll}
a_{q}=\alpha_{q} \cosh \theta_{q}+\beta_{q}^{+} \sinh \theta_{q}, & a_{q}^{+}=\alpha_{q}^{+} \cosh \theta_{q}+\beta_{q} \sinh \theta_{q}, \\
b_{q}=\alpha_{q}^{+} \sinh \theta_{q}+\beta_{q}^{+} \cosh \theta_{q}, & b_{q}^{+}=\alpha_{q} \sinh \theta_{q}+\beta_{q}^{+} \cosh \theta_{q},
\end{array}
$$

where $\alpha_{q}^{+}, \beta_{q}^{+}$are Bogoliubov's boson creation operators, the diagonal elements of the microscopic Hamiltonian, Eq. 20, can be found. On the other hand, it is known 
that the diagonal elements of the Hamiltonian $H$ are the required eigenvalues $E(q)$ of the excitation energy. In this way, the equation describing the magnon excitation energy was obtained

$$
E(q)=\sqrt{[A(q)]^{2}-[2 B(q)]^{2}}
$$

where $A(q)$ is expressed by Eq. 22 and $B(q)$ is expressed by Eq. 23 .

\subsection{Discussion of Magnon Excitations for BFO}

Based on the model described above, the macroscopic lattice displacement and the frequency of magnon excitations were calculated.

A literature value of the spring constant was used in the calculation of the macroscopic displacement for multiferroic BFO. Assuming that the spring constant for this type of multiferroic materials is $\kappa \sim 100 \mathrm{eV} \cdot \mathrm{nm}^{-2}$ [16], and using Eq. 6, the obtained value of the macroscopic lattice displacement along the $x$-direction is equal to $u_{x} \approx 0.01 \mathrm{pm}$. Another physical quantity, which can be calculated on the basis of the above model, is the frequency of magnon excitations.

To check the validity of the assumption of this model, namely, that magnon excitations are responsible for absorption for terahertz frequencies $[9,10]$, the frequency of magnon excitations was calculated. Using the well-known dependence $E=h v$ and Eq. 26, the frequency of spin wave excitations (v), i.e., the magnon frequency was determined.

Further calculations were performed based on literature data such as the exchange energy, which was taken as $J \approx 4.18 \mathrm{meV}[16,17]$, and the spin-lattice coupling, which was taken as $\lambda \approx 10 \mathrm{meV} \cdot \mathrm{nm}^{-1}$ [18], and the theoretical model described above. Calculations of the frequency of spin wave excitations were performed for the magnon wave vector $q \approx Q$.

The frequency of magnon excitations, calculated on the basis of the presented model, is $v \approx 2 \times 10^{12} \mathrm{~Hz}$, i.e., the terahertz frequency.

This result is consistent with literature experimental data $[9,10]$, and this means that magnon excitations can be responsible for the absorption, which was found for BFO.

\section{Conclusions}

The spin wave theory applied to Katsura's model gave a satisfactory result. On the basis of the foregoing, it can be concluded that the spin wave excitations are the mechanism that causes the terahertz absorption. Therefore, the model presented above helped to explain the absorption mechanism that occurs in multiferroic materials. On the basis of considerations presented in this article, it can be presumed that the spin wave excitations (i.e., magnons) are responsible for the terahertz absorption, which was experimentally measured for the multiferroic BFO $[9,10]$. 
Acknowledgment This study was financed by the Polish Ministry of Science and Higher Education under project No. N R15 000504.

Open Access This article is distributed under the terms of the Creative Commons Attribution Noncommercial License which permits any noncommercial use, distribution, and reproduction in any medium, provided the original author(s) and source are credited.

\section{References}

1. Y. Tokura, Science 312, 1481 (2006)

2. T. Kimura, T. Goto, H. Shintani, K. Ishizaka, T. Arima, Y. Tokura, Nature (London) 426, 55 (2003)

3. D. Lebeugle, D. Colson, A. Forget, M. Viret, P. Bonville, J.F. Marucco, S. Fusil, Phys. Rev. B 76, 024116 (2007)

4. A.K. Pradhan, K. Zhang, D. Hunter, J.B. Dadson, G.B. Loutts, J. Appl. Phys. 97, 093903 (2005)

5. D.F. Khozeev, A.V. Zalessky, A.A. Gippius, E.N. Morozowa, A.A. Bush, Physica B 329-333, 884 (2003)

6. M. Cazayous, Y. Gallais, A. Sacuto, Phys. Rev. Lett. 101, 037601 (2008)

7. M.K. Singh, R.S. Katiyar, J.F. Scott, J. Phys.: Condens. Matter 20, 252203 (2008)

8. R.K. Mishra, D.K. Pradhan, R.N.P. Choudhary, A. Banerjee, J. Phys.: Condens. Matter 20, 045218 (2008)

9. S. Kamba, D. Nuzhnyy, M. Savinov, J. Šebek, J. Petzelt, J. Prokleška, R. Haumont, J. Kreisel, Phys. Rev. B 75, 024403 (2007)

10. D. Talbayev, S. Lee, S.W. Cheong, A.J. Taylor, Appl. Phys. Lett. 93, 212906 (2008)

11. H. Katsura, A.V. Balatsky, N. Nagaosa, Phys. Rev. Lett. 98, 027203 (2007)

12. J.E. Hirsch, S. Tang, Phys. Rev. B 40, 4769 (1989)

13. J. Merino, R.H. McKenzie, J.B. Marston, C.H. Chung, J. Phys.: Condens. Matter 11, 2965 (1999)

14. T. Holstein, H. Primakoff, Phys. Rev. 58, 1098 (1940)

15. I.A. Sergienko, E. Dagotto, Phys. Rev. B 73, 094434 (2006)

16. J. Park, J.G. Park, G.S. Jeon, H.Y. Choi, C. Lee, W. Jo, R. Bewley, K.A. McEwen, T.G. Perring, Phys. Rev. B 68, 104426 (2003)

17. S. Ju, T.Y. Cai, G.Y. Guo, J. Chem, Phys. 130, 214708 (2009)

18. J.B. Neaton, C. Ederer, U.V. Waghmare, N.A. Spaldin, K.M. Rabe, Phys. Rev. B 71, 014113 (2005) 